\title{
INVARIANT SUBSPACE THEOREMS FOR AMENABLE GROUPS
}

\author{
by A. T. LAU, A. L. T. PATERSON and J. C. S. WONG* \\ (Received 1st February 1988)
}

\section{Introduction}

In [5], Ky Fan proved the following remarkable amenability "invariant subspace" theorem:

Let $G$ be an amenable group of continuous, invertible linear operators acting on $a$ locally convex space $E$. Let $H$ be a closed subspace of finite codimension $n$ in $E$ and $X \subset E$ be such that:

(i) $H$ and $X$ are $G$-invariant;

(ii) $(e+H) \cap X$ is compact convex for all $e \in E$;

(iii) $X$ contains an $n$-dimensional subspace $V$ of $E$. Then there exists an n-dimensional subspace of $E$ contained in $X$ and invariant under $G$.

We will refer to this result as "Ky Fan's Theorem".

The origins of this theorem seem to lie in an elementary property of Lorentz transformations. Let $T$ be a linear Lorentz transformation: so $T$ preserves the quadratic form $\langle\mathbf{x}, \mathbf{x}\rangle=x^{2}+y^{2}+z^{2}-c^{2} t^{2}$ on $\mathbb{R}^{4}$. Then there exists a three-dimensional subspace $V$ of $\mathbb{R}^{4}$ which is $T$-invariant and is positive (in the sense that $\langle\mathbf{x}, \mathbf{x}\rangle \geqq 0$ for all $\mathbf{x} \in V$ ). (For lack of a reference, we sketch the proof. Suppose firstly that $x \rightarrow T \mathbf{x}=\mathbf{x}^{\prime}$ is of the form: $x^{\prime}=\gamma(x-v t), y^{\prime}=y, z^{\prime}=z$ and $t^{\prime}=\gamma\left(t-v x / c^{2}\right)$ where $\gamma=\left(1-v^{2} / c^{2}\right)^{-1 / 2}$. Then we can take $V$ to be the subspace given by: $x=c t$. It easily follows that the desired result is true when $T$ is a boost, i.e. there exists a spatial rotation or reflection $A$ such that $A T A^{-1}$ is of the above form ([1, p. 28]). Finally, the result follows for general $T$, since such a $T$ can be decomposed into a boost followed by either a time-reversal transformation $(x, y, z, t) \rightarrow(x, y, z,-t)$ and/or a spatial orthogonal transformation $([1, \mathrm{p} .28])$.

Infinite-dimensional versions of this result were established in the work of Pontrjagin, Iohvidov, Krein and Naimark (see $[17,7,9,13,14]$ ) on " $\pi_{n}$-spaces". Indeed, let $n \geqq 1$

\footnotetext{
* The authors are grateful to the Edinburgh Mathematical Society for Centenary Fund support for a visit by the third author to Scotland in 1986
} 
and $\langle$,$\rangle be the indefinite form on l_{2}$ given by: $\langle x, y\rangle=\sum_{i=1}^{n} x_{i} \bar{y}_{i}-\sum_{n+1}^{\infty} x_{i} \bar{y}_{i}$ $\left(x=\left\{x_{i}\right\}, y=\left\{y_{i}\right\}\right)$. Naimark [14] showed: if $G$ is a commutative group of invertible, continuous, linear $\langle$,$\rangle -preserving operators on l_{2}$, then there exists a positive, $n$ dimensional subspace $V$ of $l_{2}$ which is invariant for every $T \in G$. In particular, for $T \in G$, $\left.T\right|_{V}$ is essentially a unitary operator on a finite-dimensional Euclidean space, and so Naimark's result provides useful information about the spectrum of $T$ on $l_{2}$.

In order to relate Naimark's Theorem to that of Ky Fan, we can take $E=l_{2}$ and $X=\left\{x \in l_{2}:\langle x, x\rangle \geqq 0\right\}$. However, there is no mention of an $H$ in Naimark's result. (The authors do not know for which classes of groups $G$, Naimark's Theorem holds.) The introduction of $H$ is due to Ky Fan [4], who proved a version of Naimark's Theorem when $G$ is generated by a single element. In this result, the subspace $H$ is not required to be invariant.

Ky Fan's Theorem (of the first paragraph) removes the commutative and Hilbert space restrictions of Naimark's Theorem, replacing these by amenability and the conditions involving $H$.

Ky Fan's Theorem raises a number of natural issues which the present paper will discuss.

(1) In view of the number of hypotheses in its statement, it is clearly desirable to have available a stock of (hopefully natural) examples to which the theorem applies. Ky Fan gives an infinite-dimensional example in [5], and we will develop this further in Section 6. However, there are many very natural finite-dimensional examples: these arise from symmetry properties of elementary groups. Two such examples are discussed in detail in Section 5 .

(2) The Theorem recalls Day's famous Fixed-Point Theorem (see $[6,15,16])$. However, Day's Theorem and its concomitants all involve an affine group action on a compact convex set, and a priori, it is not clear how one can regard a family of $n$ dimensional subspaces of $E$ as being compact or convex! Condition (ii) suggests that Day's Theorem is used somewhere in the proof of Ky Fan's Theorem, and indeed Ky Fan does apply Day's result to a certain set $C$ of maps defined on $E / H$. We will give two other concrete realisations of $C$, which, we think, clarify the situation and are helpful for removing the finite-dimensionality condition. The first realisation of $C$ is as a set of projections $P: E \rightarrow X$ with kernel $H$, while the second is as a subset of $H^{n}$, where $H^{n}$ has a natural $G$-action.

(3) We will show (see (4.2)) that the finite-dimensionality requirement in Ky Fan's Theorem can be removed, the $n$-dimensionality of $V$ being replaced by: $V$ is $a$ complement of $H$ in $X$. Curiously enough, the topological requirements are minimal-for example, the space $H$ need not be closed.

(4) What about the converse to Ky Fan's Theorem? Following Lau [10], let us say that $G$ has Property $P_{n}$ (for fixed $n \geqq 1$ ) if the conclusion of Ky Fan's Theorem holds. So Ky Fan's Theorem says: if $G$ is amenable then $P_{n}$ holds for all $n$. Lau showed: $G$ is amenable if and only if $P_{i}$ holds. We will show that: if, for some $n, P_{n}$ holds, then $G$ is amenable, so that $P_{m}$ is equivalent to $P_{n}$ for all $m, n \geqq 1$. More generally, (4.3) deals with the case where the co-dimension of $H$ is an arbitrary cardinal. 
(5) What about a topological version of Ky Fan's Theorem in which $G$ is a locally compact group? Some work in this direction has been done by Lau [10] and Lau and Wong $[11,12]$. We develop this theme in a different direction without finitedimensionality restrictions, the main novelty being that the map $e \rightarrow(e+H) \cap X$ is required to be "continuous" at 0 . The latter condition is satisfied in all reasonable cases.

It will be found useful to replace a " $K y$ Fan system" $(E, H, X, G)-$ a quadruple satisfying a natural version of (i), (ii) and (iii)-by a reduced $K y$ Fan system. In the latter case $X=\cup X_{H}$, where $X_{H}$ is the set of $H$-complements in $X$. We show in Section 2 that questions about Ky Fan's Theorem can (normally) be treated by looking at the reduced case. Intuitively, a general Ky Fan system is just a reduced one with compact convex "blobs" stuck onto $X$.

The inspiration for much of the theory presented here came from considering geometrical examples, and the reader may well find it helpful to have Examples 1 and 2 of Section 5 in mind when reading the paper.

\section{Ky Fan and reduced Ky Fan systems}

Let $\mathbb{F}$ be either $\mathbb{R}$ or $\mathbb{C}, E$ be a locally convex space over $\mathbb{F}$, and $H$ be a subspace of $E$. The subspace $H$ need not be closed in $E$. A subspace $V$ of $E$ is said to be complementary to $H$ if $V+H=E, V \cap H=E$. Of course, by Zorn's lemma, $H$ always admits a complementary subspace $V$, and the dimension of $V$ (the cardinality of a Hamel basis for $V$ ) is independent of $V$. The family of subspaces of $E$ complementary to $V$ is denoted by $\mathscr{C}_{H}(E)$ or simply by $\mathscr{C}_{H}$. If $X \subset E$, then

$$
X_{H}=\left\{V: V \subset X, V \in \mathscr{C}_{H}\right\}
$$

The algebra of continuous linear transformations of $E$ is denoted by $B(E)$.

A quadruple $(E, H, X, G)$, with $E, H$ as above, is called a $K y$ Fan system if:

(i) $X \subset E$ is such that

(a) $(e+H) \cap X$ is compact convex for all $e \in E$,

(b) $X_{H} \neq \emptyset$,

and

(ii) $G$ is a group of invertible elements of $B(E)$ such that

(a) $x X=X$ for all $x \in G$,

(b) $x H=H$ for all $x \in G$.

Given such a Ky Fan system $(E, H, X, G)$, let $m$ be the dimension of a complementary subspace $V$ for $H$ in $E$. Ky Fan [5] introduced a more general system when $m$ is finite. $\mathrm{Ky}$ Fan assumes that $H$ is closed in $E$, and replaces (ii)(a) by the weaker requirement that $x X_{H}=X_{H}$ for all $x \in G$. However, we have preferred the more natural (ii)(a), though in fact the results of this paper hold with minor modifications when (ii)(a) is replaced by Ky Fan's original assumption. Ky Fan also allows $G$ to be a semigroup (cf. (4.8)).

The Ky Fan system $(E, H, X, G)$ is called reduced if $X=\cup X_{H}$. Reduced systems are 
geometrically "nicer" than general systems, and as we shall see, problems involving Ky Fan systems usually need only be studied in the reduced case. Examples of Ky Fan systems will be discussed later in the paper, but the following very elementary example illustrates how such systems are naturally associated with reduced systems.

Consider the $\mathrm{Ky}$ Fan system $(E, H, X, G)$ where $E=\mathbb{R}^{2}, H=\mathbb{R} \times\{0\}, X$ is the region between the curves $x= \pm y^{2}$, and $G$ is trivial. Note that $\{0\} \times \mathbb{R} \in X_{H}$. In case (b), we have the same $E, H$ and $G$ but with $X=\{0\} \times \mathbb{R}$. The maximal reduced Ky Fan system "inside" $(E, H, X, G)$ is $(E, H,\{0\} \times \mathbb{R}, G)$, as is obvious from the geometry.

We will show that there is always a unique maximal reduced Ky Fan system inside a given Ky Fan system (Proposition 2.3).

Our first Proposition is easy but very important.

Proposition 2.1. Let $(E, H, X, G)$ be a $K y$ Fan system and let $V \in X_{H}$. Then:

(i) for each $e \in E,(e+H) \cap V$ is a singleton $\left\{P_{V}(e)\right\}$;

(ii) the map $P_{V}$ of (i) is a linear projection of $E$ onto $V$ and $k e r P_{V}=H$.

Proof. Since $V \oplus H=E$, the quotient map $Q_{H}: E \rightarrow E / H$ is a linear isomorphism when restricted to $V$. Hence $(e+H) \cap V \neq \emptyset$ for all $e \in E$. If $e+h_{1}, e+h_{2} \in(e+H) \cap V$, then $\left(h_{1}-h_{2}\right) \in(0+H) \cap V$, and since $H, V$ are subspaces, $\mathbb{F}\left(h_{1}-h_{2}\right) \subset(0+H) \cap V$, a compact set. Hence $h_{1}=h_{2}$ and (i) follows. Note also that $H \cap V=\{0\}$. We now prove (ii). Let $e_{1}, e_{2} \in E, \lambda_{1}, \lambda_{2} \in \mathbb{F}$. Since $V$ is a vector space $\lambda_{1} P_{V}\left(e_{1}\right)+\lambda_{2} P_{V}\left(e_{2}\right) \in V$. But we also have

$$
\lambda_{1} P_{V}\left(e_{1}\right)+\lambda_{2} P_{V}\left(e_{2}\right) \in \lambda_{1}\left[\left(e_{1}+H\right) \cap V\right]+\lambda_{2}\left[\left(e_{2}+H\right) \cap V\right]=\left(\left(\lambda_{1} e_{1}+\lambda_{2} e_{2}\right)+H\right) \cap V .
$$

Hence $P_{V}$ is linear using (i). Again by (i), $P_{V}^{2}=P_{V}$. Finally, since $H \cap V=\{0\}$, we have $P_{V}(H)=\{0\}$, and if $(e+H) \cap V=\{0\}$, then $0 \in e+H$ and $e \in H$. So ker $P_{V}=H$.

Let $\mathscr{L}(E)$ be the algebra of linear transformations of $E$. The elements of $\mathscr{L}(E)$ are not assumed to be continuous. Now let $H$ be a subspace of $E$ and define

$$
\mathscr{P}_{H}=\left\{P \in \mathscr{L}(E): P^{2}=P, \operatorname{ker} P=H\right\}
$$

The study of $\mathscr{P}_{H}$ is motivated by (2.1): the map $P_{V}$ of $(2.1)$ belongs to $\mathscr{P}_{H}$.

We give $\mathscr{L}(E)$ the pointwise topology which it inherits as a subset of $E^{E}$ : so a net $T_{\delta} \rightarrow T$ in $\mathscr{L}(E)$ if and only if $T_{\delta} e \rightarrow T e$ for all $e \in E$. Clearly, $\mathscr{L}(E)$ is a locally convex space.

Proposition 2.2. The set $\mathscr{P}_{H}$ is a convex, left zero sub-semigroup of $\mathscr{L}(E)$, and is closed in $\mathscr{L}(E)$ if $H$ is closed in $E$.

Proof. Let $P, Q \in \mathscr{P}_{H}, e \in E$. Since $Q(e-Q(e))=0$, we have $e-Q(e) \in H=$ ker $P$. So $P(e-Q(e))=0$ and $P=P Q$. So $\mathscr{P}_{H}$ is a left zero subsemigroup of $\mathscr{L}(E)$. Now let $\alpha \in[0,1], R=\alpha P+(1-\alpha) Q$. Since $\mathscr{P}_{H}$ is a left zero semigroup, we have $R^{2}=\alpha^{2} P+$ 
$\alpha(1-\alpha)(P+Q)+(1-\alpha)^{2} Q=\alpha P+(1-\alpha) Q=R$. Further, $\operatorname{Re} \in(\alpha e+H)+((1-\alpha) e+H)=e+$ $H$ and $H \subset \operatorname{ker} R$. It follows that $\operatorname{ker} R=H$. So $R \in \mathscr{P}_{H}$, and $\mathscr{P}_{H}$ is convex.

Finally, suppose that $H$ is closed in $E$ and $\left\{P_{\delta}\right\}$ is a net in $\mathscr{P}_{H}$ converging to $T \in \mathscr{L}(E)$. Then $H \subset \operatorname{ker} T$ and $T e \in e+H$ for all $e \in E$. It follows that $T \in \mathscr{P}_{H}$ and so $\mathscr{P}_{H}$ is closed.

Proposition 2.3. Let $(E, H, X, G)$ be a KyFan system and let $Y=\cup X_{H}$. Then $(E, H, Y, G)$ is a reduced $K y$ Fan system.

Proof. (i) $(a)$ is the only non-trivial condition to be checked. Let $e \in E$ and $C_{e}=$ $(e+H) \cap X, D_{e}=(e+H) \cap Y$. We show first that $D_{e}$ is convex. Let $e_{1}, e_{2} \in D_{e}$ and $\alpha \in[0,1]$. By (2.1), there exist $P_{1}, P_{2} \in \mathscr{P}_{H}$ with $P_{i}(E) \in X_{H}$ and $P_{i}(e)=e_{i}$. By (2.2), $P=\alpha P_{1}+(1-\alpha) P_{2} \in \mathscr{P}_{H}$. For any $f \in E$, the set $C_{f}$ is convex and contains $P_{1}(f), P_{2}(f)$ and hence also contains $P(f)=\alpha P_{1}(f)+(1-\alpha) P_{2}(f)$. So $P(E) \subset X$. Since $P \in \mathscr{P}_{H}$, we also have that $P(E)=V \in X_{H}$. So $\alpha e_{1}+(1-\alpha) e_{2}=P(e) \in V \subset Y$, and $D_{e}$ is convex.

We now show that $D_{e}$ is compact. Let $\left\{e+h_{\delta}\right\}$ be a net in $D_{e}$. Since $C_{e}$ is compact, we can suppose that $h_{\delta} \rightarrow h \in H$. By the preceding paragraph, there exists a net $\left\{P_{\delta}\right\}$ in $\mathscr{P}_{H}$ such that $P_{\delta}(E) \in X_{H}$ and $P_{\delta}(e)=e+h_{\delta}$. We can regard $\left\{P_{\delta}\right\}$ as a net in the set $\prod_{\delta \in E} C_{\delta}$, which is compact in the product topology by Tychonoff's Theorem. Replacing $\left\{P_{\delta}\right\}$ by a subnet, we can suppose that $\left\{\mathrm{P}_{\delta} f\right\}$ converges for all $f \in E$. So $P_{\delta} \rightarrow P$ for some $P \in \mathscr{L}(E)$, and it is easily checked that $P \in \mathscr{P}_{H}$ and $P(E) \in X_{H}$. So $e+h_{\delta}=P_{\delta}(e) \rightarrow P e \in D_{e}$, and $D_{e}$ is compact.

Corollary 2.4. The following are equivalent for any group $G$ and cardinal $m$.

(i) There exists a $G$-invariant subspace $Z \in X_{H}$ for every $K y$ Fan system $(E, H, X, G)$ with $\operatorname{dim} E / H=m$.

(ii) There exists a $G$-invariant subspace $Z \in X_{H}$ for every reduced $K y$ Fan system $(E, H, X, G)$ with $\operatorname{dim} E / H=m$.

Proof. It is trivial that (i) implies (ii). Suppose, then, that (ii) holds and let $(E, H, X, G)$ be a $\mathrm{Ky}$ Fan system with $\operatorname{dim} E / H=m$. Now apply (ii) to the reduced Ky Fan system $(E, H, Y, G)$ of (2.3) to obtain a $G$-invariant subspace $Z \in Y_{H} \subset X_{H}$. So (ii) implies (i).

Note. The reduced Ky Fan system $(E, H, Y, G)$ of $(2.3)$ is, in a natural way, the "largest" reduced Ky Fan system "contained in" $(E, H, X, G)$.

\section{Reduced Ky Fan systems as compact convex sets}

In this section, we will show that a reduced Ky Fan system can be regarded $(a)$ as a compact, convex set of projection operators, or $(b)$ as a compact convex subset of a product space $H^{m}$. The two formulations are, of course, closely related, but both points of view are useful. 
Let $G$ be a group of invertible elements of $B(E), H$ be a $G$-invariant subspace of $E$ and $\mathscr{P}_{H}$ be given the pointwise topology as in Section 2 . We obtain a natural left action of $G$ on $\mathscr{P}_{H}$ by setting

$$
(x P)(e)=x P\left(x^{-1} e\right)
$$

$\left(x \in G, P \in \mathscr{P}_{H}, e \in E\right)$. Indeed, for such $x$ and $P$,

$$
(x P)^{2}(e)=(x P)\left(x P\left(x^{-1} e\right)\right)=x\left[P\left(x^{-1} x P\left(x^{-1} e\right)\right)\right]=x P\left(x^{-1} e\right)=(x P)(e),
$$

so that $(x P)^{2}=x P$. Further, since $x H=H$, it follows that $\operatorname{ker}(x P)=H$, so that $x P \in \mathscr{P}_{H}$. It is easily checked that the map $(x, P) \rightarrow x P$ is a left action of $G$ on $\mathscr{P}_{H}$. Further, this action is both affine and continuous. (Recall that by (2.2), $\mathscr{P}_{H}$ is convex.)

The theorem of this section asserts that there is a natural one-to-one correspondence between reduced Ky Fan systems $(E, H, X, G)$ and $G$-invariant, compact, convex subsets of $\mathscr{P}_{H}$.

Now let $m=\operatorname{dim} E / H$ and $V$ be a subspace of $E$ complementary to $H$. Let $B$ be a Hamel basis for $V$ : so $m$ is the cardinality $|B|$ of $B$. Let $H^{B}$ be given the product topology. Then $H^{B}$ is a locally convex space. We define a left $G$-action on $H^{B}$ as follows. Let $r \in H^{B}$ and $V_{r}=\operatorname{Span}\{b+r(b): b \in B\}$. Then $V_{r}$ is a subspace of $E$ complementary to $H$, and as $H$ is $G$-invariant, $x V_{r}$ is also complementary to $H(x \in G)$. It follows that there exists a unique element $s(b) \in H \cap\left(x V_{r}-b\right)$ for each $b \in B$. We define

$$
x r=s .
$$

Note that in general, $(x r)(b) \neq x(r(b))$ for $b \in B$ (c.f. $\S 5$, Example 2). The fact that the map $(x, r) \rightarrow x r$ is a continuous, affine left action of $G$ on $H^{B}$ follows from the proof of the following theorem.

Theorem 3.1. Let $E, H, G$ and $B$ be as above. Then there exist natural one-to-one, $G$-equivariant correspondences between each of the following three sets:

(i) the set of reduced $K y$ Fan systems $(E, H, X, G)$;

(ii) the set of compact, convex, $G$-invariant subsets of $\mathscr{P}_{H}$;

(iii) the set of compact, convex, G-invariant subsets $K$ of $H^{B}$.

Proof. Let $(E, H, X, G)$ be a reduced $\mathrm{Ky}$ Fan system. For each $V \in X_{H}$, let $P_{V} \in X_{H}$ be the projection in $\mathscr{P}_{H}$ associated with $V$ (c.f. (2.1)). Let $C=\left\{P_{V}: V \in X_{H}\right\}$. From the proof of (2.3), $C$ is a compact convex subset of $\mathscr{P}_{H}$. Since, for $V \in X_{H}, x \in G, P_{x V}=x P_{V}$, it follows that $C$ is $G$-invariant. Conversely, given a compact, convex, $G$-invariant subset $D$ of $\mathscr{P}_{H}$, we obtain a reduced $\mathrm{Ky}$ Fan system by setting $X=\bigcup_{P \in D} P(E)$. This establishes the correspondence between (i) and (ii).

We now establish the correspondence between (ii) and (iii). We first define a map $\Phi: H^{B} \rightarrow \mathscr{P}_{H}$. For $r \in H^{B}$, we define, in the notation preceding the theorem, $\Phi(r)=P_{V_{r}}$. 
Since $\Phi(r)(b)=b+r(b)$, it is easily checked that $\Phi$ is affine and is bicontinuous from $H^{B}$ onto $\mathscr{P}_{H}$. We now check that $\Phi$ preserves the $G$-action. Let $b \in B, x \in G$ and set $(x r)(b)=k$. By definition, $\{k\}=\left(x V_{r}-b\right) \cap H$, so that $\left\{x^{-1} k\right\}=\left(V_{r}-x^{-1} b\right) \cap H=$ $-x^{-1} b+V_{r} \cap\left(x^{-1} b+H\right)=\left\{-x^{-1} b+P_{V_{r}}\left(x^{-1} b\right\}\right.$. Hence $k=-b+\left(x P_{V_{r}}\right)(b)$, giving $\Phi(x r)(b)=b+k=\left(x P_{V_{r}}\right)(b)$, so that $\Phi(x r)=x \Phi(r)$. Thus $\Phi$ preserves the $G$-action. Thus $\Phi$ establishes the correspondence between (ii) and (iii).

\section{Fixed-subspace theorems}

The following proposition enables one to construct new Ky Fan systems from given ones. In this result, the locally convex space $\oplus E_{\alpha}$ is not given the usual direct sum topology (c.f. [8, p. 211f.]). Instead, we give $\oplus E_{a}$ the relative topology which it inherits as a subspace of $\pi E_{\alpha}$. (The latter space has, of course, the pointwise topology.)

Proposition 4.1. If $\left\{\left(E_{\alpha}, H_{\alpha}, X_{\alpha}, G_{\alpha}\right): \alpha \in A\right\}$ is a family of reduced $K y$ Fan systems, then so also are $\left(\oplus E_{\alpha}, \oplus H_{\alpha}, \oplus X_{\alpha}, \pi G_{\alpha}\right),\left(\pi E_{\alpha}, \pi H_{\alpha}, \pi X_{\alpha}, \pi G_{\alpha}\right)$.

Proof. Let $(E, H, X, G)=\left(\oplus E_{\alpha}, \oplus H_{\alpha}, \oplus X_{\alpha}, \pi G_{\alpha}\right)$. Of course, $G$ acts on $E$ in the natural way. If $e=\sum_{i=1}^{n} e_{\alpha_{i}} \in E, e_{\alpha_{i}} \in E_{\alpha_{i}}$, then since $H_{\alpha} \cap X_{\alpha}=\{0\}$ for all $\alpha,(e+H) \cap X=$ $\left[\bigoplus_{i=1}^{n}\left(e_{\alpha_{i}}+H_{\alpha_{i}}\right) \cap X_{\alpha_{1}}\right]$. Clearly $(e+H) \cap X$ is compact and convex. If for each $\alpha$, $V_{\alpha} \in X_{H_{a}}$, then $\oplus V_{\alpha} \in X_{H}$ so that $X_{H} \neq \emptyset$. It is obvious that $x X=X, x H=H$ for all $x \in G$, and that $X=\cup X_{H}$. So $(E, H, X, G)$ is a reduced Ky Fan system.

The corresponding result for $\left(\pi E_{\alpha}, \pi H_{\alpha}, \pi X_{\alpha}, \pi G_{\alpha}\right)$ is straightforward to prove using Tychonoff's Theorem.

The next Theorem essentially generalises Ky Fan's Theorem and answers a problem raised in Lau [10]. Our theorem is valid for arbitrary cardinals $m$, so that the finitedimensional requirement needed in earlier work is not required.

Theorem 4.2. Let $G$ be a group and $m$ be a cardinal. Then (i) and (ii) are equivalent.

(i) $G$ is amenable.

(ii) For every $K y$ Fan system $(E, H, X, G)$ with $\operatorname{dim} E / H=m$, there exists a $G$-invariant subspace in $X_{H}$.

Proof. That (i) implies (ii) follows from (2.4), (3.1) and Day's Fixed-Point Theorem.

Conversely, suppose that (ii) holds. Let $V$ be a complex vector space of dimension $m$ and $B$ be a basis for $V$. Then $V=\bigoplus_{b \in B} \mathbb{C} b$. Give $V$ the relative product topology (as in (4.1)). Let $H$ be the locally convex space $l_{\infty}(G)^{*}$ with the weak* topology and let $C$ be the set of means on $G$. Then $C$ is a compact convex subset of $H$.

Let $E$ be the locally convex space $H \times V$ with the product topology. For each $c \in C$, let $V_{c}=\operatorname{Span}\{(c, b): b \in B\}$, and identify $H$ with $H \times\{0\}, V$ with $\{0\} \times V$. We claim that $E=H \oplus V_{c}$. Indeed, $H \oplus V_{c} \supset \operatorname{Span}\{(-c, 0)+(c, b): b \in B\}=V$ and so $H+V_{c} \supset H \oplus V=E$. Also, if $w=\sum_{i=1}^{n} \lambda_{i}\left(c, b_{i}\right) \in H(=H \times\{0\}), \lambda_{i} \in \mathbb{C}, b_{i} \in B$, then $\sum_{i=1}^{n} \lambda_{i} b_{i}=0$, so that $\lambda_{i}=0$ 
$(1 \leqq i \leqq n)$, and $w=0$. So $E=H \oplus V_{c}$. Let $X=\bigcup_{c \in c} V_{c}$. The group $G$ acts continuously on $E$ as follows: for $x \in G,(h, v) \in E$, we define $x(h, v)=(x h, v)$, where $G$ has the natural left action on $H=l_{\infty}(G)^{*}$. (So $x h(\phi)=h(\phi x)$ where $\phi \in l_{\infty}(G)$ and $\phi x \in l_{\infty}(G)$ is given by: $\phi x(y)=\phi(x y)(y \in G)$. Note that $x V_{c}=V_{x c}$ for all $x \in G, c \in C$. We claim that $(E, H, X, G)$ is a reduced $\mathrm{Ky}$ Fan system with $\operatorname{dim} E / H=m$. The only non-trivial condition to be checked is that $(e+H) \cap X$ is compact convex for all $e \in E$.

To this end, let $e \in E$. Then $e=\left(h_{0}, v_{0}\right)$, where $v_{0}=\sum_{i=1}^{n} \lambda_{i} b_{i}$ for some $h_{0} \in H, \lambda_{i} \in \mathbb{C}$, $b_{i} \in B$. Obviously, $(e+H) \cap X=\left(v_{0}+H\right) \cap X$. Let $z \in\left(v_{0}+H\right) \cap X$. Then $z \in V_{c_{0}}$ for some $c_{0} \in C$, so that there exist $h^{\prime} \in H, \gamma_{j} \in \mathbb{C}, b_{j}^{\prime} \in B$ such that $z=v_{0}+h^{\prime}=\sum_{j=1}^{m} \gamma_{j}\left(c_{0}, b_{j}^{\prime}\right)$. So $\sum_{i=1}^{n} \lambda_{i} b_{i}=\sum_{j=1}^{m} \gamma_{j} b_{j}^{\prime}, h^{\prime}=\left(\sum_{j=1}^{m} \gamma_{j}\right) c_{0}$. Since $B$ is a basis for $V$, we have $z=v_{0}+\left(\sum_{i=1}^{n} \lambda_{i}\right) c_{0}$. It follows that

$$
\left(v_{0}+H\right) \cap X=v_{0}+\left(\sum_{i=1}^{n} \lambda_{i}\right) C
$$

which is compact convex.

Thus we can apply (ii) to the system $(E, H, X, G)$ to obtain a $G$-invariant subspace $W \in X_{H}$. We will show that $W=V_{c}$ for some $c \in C$. Let

$$
Z=\left\{\sum_{i=1}^{n} \lambda_{i} b_{i}: \lambda_{i} \in \mathbb{C}, \sum_{i=1}^{n} \lambda_{i}=0, b_{i} \in B, n \in \mathbb{P}\right\}
$$

Clearly $Z$ is a subspace of $E$. For any $c \in C$, a typical element of $V_{c}$ is of the form $\sum_{i=1}^{m} \mu_{i} b_{i}+\left(\sum_{i=1}^{m} \mu_{i}\right) c\left(\mu_{i} \in \mathbb{C}\right)$, and so $Z \subset V_{c}$. Let $w_{1} \in W$. Since $W \subset X=\bigcup_{c \in c} V_{c}$, we can find a $c_{1}$ such that $w_{1}$ is of the form $\sum_{i=1}^{m} \mu_{i} b_{i}+\left(\sum_{i=1}^{m} \mu_{i}\right) c_{1}$. If $\sum_{i=1}^{m} \mu_{i}=0$, then $w_{1} \in Z$. Suppose now that $\sum_{i=1}^{m} \mu_{i} \neq 0$. We claim that $c_{1}$ is independent of $w_{1}$. Indeed, suppose that $w_{2} \in\left(W \cap V_{c_{2}}\right) \sim Z$ for some $c_{2} \in C$. Then we can write $w_{2}=\sum_{j=1}^{p} v_{j} b_{j}^{\prime}+\left(\sum_{j=1}^{p} v_{j}^{\prime}\right) c_{2}$ where $\sum_{j=1}^{p} v_{j}^{\prime} \neq 0$ and $b_{j}^{\prime} \in B$. After scaling $w_{1}$ and $w_{2}$ (if necessary), we can suppose that $\sum_{j=1}^{p} v_{j}=1=\sum_{i=1}^{m} \mu_{i}$. Then $W$ is a subspace contained in $X$, and we have, using (1),

$$
\left(w_{1}-w_{2}\right) \in X \cap\left(\sum_{i=1}^{m} \mu_{i} b_{i}-\sum_{j=1}^{p} v_{j}^{\prime} b_{j}^{\prime}+H\right)=\left\{\sum_{i=1}^{m} \mu_{i} b_{i}-\sum_{j=1}^{p} v_{j}^{\prime} b_{j}^{\prime}\right\} \subset Z .
$$

Since the $H$-component of $w_{1}-w_{2}$ is $c_{1}-c_{2}$ and $Z \subset V$, or, more precisely, $\{0\} \times V$, it follows that $c_{1}=c_{2}$. So for some $c, W \subset \mathrm{V}_{c}$ and since $V_{c}$ is also complementary to $H$, we have $W=V_{c}$ for some $c \in C$.

Since $W$ is $G$-invariant, we have $V_{c}=x V_{c}=V_{x c}$ for all $x \in G$, so that $x c=c$ for all $x \in G$. Hence $c$ is a left invariant mean on $G$, and $G$ is amenable.

Corollary 4.3. Let $G$ be a group and $m, n$ be cardinals $>0$. Then (i) and (ii) are equivalent:

(i) There exists a $G$-invariant subspace in $X_{H}$ for every $K y$ Fan system $(E, H, X, G)$ with $\operatorname{dim} E / H=m$. 
(ii) There exists a G-invariant subspace in $X_{H}$ for every $K y$ Fan system $(E, H, X, G)$ with $\operatorname{dim} E / H=n$.

The preceding Corollary does not explicitly involve the amenability of $G$. We know of no proof of (4.3) which does not use the amenability criterion of (4.2).

The next two results are generalisations of results of Ky Fan $[3,4]$-see also Naimark [11] and Section 1.

Theorem 4.4. Let $V, H$ be Banach spaces with $H$ reflexive and $E$ be the Banach space direct sum $V \oplus H$. Let $q: E \rightarrow \mathbb{R}$ be given by: $q(v+h)=\|v\|-\|h\|(v \in v, h \in H)$. Let $G$ be $a$ discrete, amenable group of invertible elements of $B(E)$ such that:

(i) $x H=H$ for all $x \in G$;

(ii) $q(x e) \geqq 0$ whenever $q(e) \geqq 0(x \in G, e \in E)$.

Then there exists a complementary subspace $W$ for $H$ in $E$ such that:

(a) $x W=W$ for all $x \in G$;

(b) $q(e) \geqq 0$ for all $e \in E$.

Proof. Let $X=\{e \in E: q(e) \geqq 0\}$ and give $E$ the weak topology. For each $e \in V$, the set $C_{e}=(e+H) \cap X=\{e+h: h \in H,\|h\| \leqq\|e\|\}$ is obviously convex, and since $H$ is reflexive, $C_{e}$ is also compact. It follows that $C_{e}$ is compact convex for all $e \in E$. Clearly, $V \in X_{H}$ and by (ii), $X$ is $G$-invariant. So $(E, H, X, G)$ is a $\mathrm{Ky}$ Fan system, and the existence of $W$ follows from (4.3).

Corollary 4.5. Let $K$ be a closed subspace of a Hilbert space $\mathscr{H}$ and $H=K^{\perp}$. Let $G$ be an amenable group of invertible elements of $B(\mathscr{H})$ which leave $H$ invariant and which preserve the form $\langle$,$\rangle of \mathscr{H}$, where

$$
\left\langle k+h, k^{\prime}+h^{\prime}\right\rangle=\left\langle k, k^{\prime}\right\rangle-\left\langle h . h^{\prime}\right\rangle \quad\left(k, k^{\prime} \in K, h, h^{\prime} \in H\right) .
$$

Then there exists a G-invariant, complementary subspace $W$ for $\boldsymbol{H}$ in $E$ such that $\langle w, w\rangle \geqq 0$ for all $w \in W$.

It is well-known that amenability for a locally compact group $G$ is equivalent to a topological fixed-point theorem. It is natural to enquire if there is a topological version of (4.2). To answer this, we require the notion of a topological Ky Fan system. The main novelty is the "continuity at 0 " requirement of $(d)$ below. Most "naturally occurring" examples of Ky Fan systems are, in fact, topological.

In connection with $(c)$, recall (c.f. $[8, \S 10.7])$ that if $H$ is a closed subspace of a locally convex space $E$, then a topological complement for $H$ in $E$ is a closed subspace $V$ of $E$ such that the map $(v, h) \rightarrow v+h$ is a topological isomorphism from $V \times H$ onto $E$. (Of course, if $E$ is Fréchet, then being a topological complement for $H$ is equivalent to being a closed complementary subspace for $H$. In general, this equivalence is not true.) 
Definition. A topological Ky Fan system is a Ky Fan system $(E, H, X, G)$ such that:

(a) the action of $G$ is separately continuous on $E$ and jointly continuous on $X$;

(b) $H$ is a closed subspace of $E$;

(c) $X$ contains a topological complement for $H$ in $E$;

(d) the map $e \rightarrow C_{e}=(e+H) \cap X$ is continuous at 0 , i.e. given a neighbourhood $U$ of 0 in $E$, there exists a neighbourhood $U_{1}$ of 0 in $E$ such that $C_{e} \subset C_{0}+U$ for all $e \in U_{1}$.

Note. It would, perhaps, be more natural to require the $G$-action on $E$ to be jointly continuous on $E$. Unfortunately, our Theorem 4.7 depends crucially on using a topological Ky Fan system for which the $G$-action on $E$ is not jointly continuous. We do not know if Theorem 4.7 is still true when (a) is replaced by the stronger joint continuity condition.

Let $(E, H, X, G)$ be a topological $\mathrm{Ky}$ Fan system. The set of topological complements $V$ of $H$ with $V \subset X$ is denoted (with a slight abuse of notation) by $X_{H}$. The quadruple $(E, H, X, G)$ is called reduced if $X=\cup X_{H}$.

Proposition 4.6. The quadruple $(E, H, Y, G)$ where $Y=\cup X_{H}$, is a reduced topological Ky Fan system.

Proof. Of course, this is the topological version of (2.3). We will be content to sketch the modifications in the proof of (2.3) required.

Let $\mathscr{P}_{H}^{t}=\left\{P \in B(E): P^{2}=P\right.$, $\left.\operatorname{ker} P=H\right\}$. Then (c.f. (2.2)) $\mathscr{P}_{H}^{t}$ is a convex, left zero semigroup. Now follow the first part of the proof of (2.3) with $\mathscr{P}_{H}$ replaced by $\mathscr{P}_{H}^{t}$. Let $K=\left\{P \in \mathscr{P}_{H}^{i}: P(E) \in X_{H}\right\}$. We then have to show that if $\left\{P_{\delta}\right\}$ is a net in $K$ and $P_{\delta} \rightarrow P$ in $\mathscr{L}(E)$, then $P \in K$. To this end, let $U$ be a neighbourhood of 0 in $E$. By (d) above, there exists a neighbourhood $U_{1}$ of 0 in $E$ such that $C_{e} \subset C_{0}+U$ for all $e \in U_{1}$. Since $C_{e}$ is compact, $P_{\delta}(e) \in C_{e}$ for all $\delta$, and as $P_{\delta} \rightarrow P$, we have $P(e) \in C_{0}+U$ for all $e \in U_{1}$. It follows that $P$ is continuous. For otherwise, there would exist a net $e_{\sigma} \rightarrow 0$ in $E$ and a continuous seminorm $p$ on $E$ such that $p\left(P e_{\sigma}\right) \geqq 1$ for all $\sigma$. Let $U=p^{-1}([0,1])$ and $U_{1}$ be as in (d). Then, using the $e_{\sigma}$, we can find a sequence $\left\{f_{n}\right\}$ in $U_{1}$ with $n f_{n} \in U_{1}$, $p\left(P f_{n}\right) \geqq 1$. But this means that $\left\{p\left(P\left(n f_{n}\right)\right)\right\}$ is an unbounded sequence in the bounded set $p\left(C_{0}+U\right)$, and we derive a contradiction. So $P$ is continuous as required. It follows that $P(E)$ is a topological complement for $H$, and so belongs to $X_{H}$. So $P \in K$. It then follows as in (2.3) that $D_{e}=(e+H) \cap Y=\{P(e): P \in K\}$ is compact convex for all $e \in H$. It remains to show that $e \rightarrow D_{e}$ is continuous at 0 . To this end, let $V$ be a circled neighbourhood of 0 in $E$ with $V+V \subset U$. Let $V_{1}$ be a neighbourhood of 0 in $E$ such that $C_{e} \subset C_{0}+V$ for all $e \in V_{1}$. Let $P \in K$. Then $P e \in C_{0}+V\left(e \in V_{1}\right)$. Let $r \in(0,1)$ be such that $r C_{0} \subset V$ and $W_{1}=r V_{1}$. Then if $w \in W_{1}, w=r e$, we have $P(w)=P(r e) \in r C_{0}+r V \subset V+V \subset U$. So $D_{w} \subset$ $U\left(=D_{0}+U\right)$ whenever $w \in W$, and $e \rightarrow D_{e}$ is continuous at 0 .

Theorem 4.7. Let $G$ be a locally compact group. Then $G$ is amenable if and only if for 
every topological $K y$ Fan system $(E, H, X, G)$, there exists a G-invariant topological complement for $H$ in $X$.

Proof. Suppose that the last condition of the theorem holds. We will show that $G$ is amenable. Let $U_{r}(G)$ be the space of right uniformly continuous functions on $G$ : so $U_{r}(G)$ consists of continuous bounded functions $\phi: G \rightarrow \mathbb{C}$ such that the map $x \rightarrow \phi x$ is sup-norm continuous. (Recall that $\phi x(y)=\phi(x y)$ for all $y \in G$.) We now modify an argument of Lau [10]. Let $E=U_{r}(G)^{*}$ with the weak* topology, $H=\{\alpha \in E: \alpha(1)=0\}, C$ be the set of means (states) on $U_{r}(G)$ and $X=\cup\{\mathbb{C} m: m \in C\}$. We claim that $(E, H, X, G)$ is a (reduced) topological Ky Fan system. Firstly, for $e \in E$, the set $C_{e}=(e+H) \cap X=$ $e(1) C$ is a compact convex set. Now let $U$ be a neighbourhood of 0 in $E$. Since $C$ is compact, there exists $k>0$ such that $a C \subset U$ for $0 \leqq|a|<k$. If $U_{1}=\{e \in E:|e(1)|<k\}$, then $C_{e}=e(1) C \subset 0+U=C_{0}+U$ for all $e \in U_{1}$. So the map $e \rightarrow C_{e}$ is continuous. It is easy and well known that the action of $G$ on $E$ is separately continuous. (The action is jointly continuous on bounded sets.) We now show that the action is jointly continuous on $X$. Indeed, suppose that nets $x_{\delta} \rightarrow x$ in $G$ and $e_{\delta} \rightarrow e$ in $X$. We can write $e_{\delta}=\lambda_{\delta} m_{\delta}, e=\lambda m$ for $\lambda_{\delta}, \lambda \in \mathbb{C}, m_{\delta}, m \in C$. Then $\lambda_{\delta} m_{\delta}(1) \rightarrow \lambda m(1)$, so that $\lambda_{\delta} \rightarrow \lambda$. It follows that eventually $\left\{\lambda_{\delta} m_{\delta}\right\}$ is inside a bounded set, and so $x_{\delta} e_{\delta} \rightarrow x e$ as required. It now follows that $(E, H, X, G)$ is a topological Ky Fan system, the remaining verifications being trivial.

By hypothesis there exists a mean $\mathbb{C} m$ such that $m$ is $G$-invariant. It follows that $m$ is a left invariant mean on $U_{r}(G)$ so that $G$ is amenable.

Conversely, suppose that $G$ is amenable and let $(E, H, X, G)$ be a topological Ky Fan system. By (4.6), we can suppose that the system is reduced. Let $K=\left\{P \in \mathscr{P}_{H}^{t}: P(E) \subset X\right\}$ as in (4.6). Then $K$ is a $G$-invariant, compact, convex subset of $\mathscr{L}(E)$ and $K \subset B(E)$. We claim that the $G$-action on $K$ is separately continuous. Indeed, as earlier, the map $P \rightarrow x P$ on $K$ is continuous for each $x \in G$. If $x_{\delta} \rightarrow x$ in $G$ and $P \in K, e \in E$, then, since $P$ is continuous, we have $P\left(x_{\delta}^{-1} e\right) \rightarrow P\left(x^{-1} e\right)$ in $X$. Since the action of $G$ on $X$ is jointly continuous, it follows that $x_{\delta} P\left(x_{\delta}^{-1} e\right) \rightarrow x P\left(x^{-1} e\right)$, so that $x_{\delta} P \rightarrow x P$. Since $G$ is amenable, there exists a $G$-fixed point $P$ in $K$. Then $V=P(E)$ is a $G$-invariant topological complement for $H$ in $X$.

Note. Let $N$ be a closed subgroup of $G$. The homogeneous space $G / N$ is called amenable (c.f.[2]) if there exists a left $G$-invariant mean on the space of bounded continuous functions $\phi: G / N \rightarrow \mathbb{C}$. Using a fixed-point theorem of Eymard, we can prove the following extension of (4.7): the homogeneous space $G / N$ is amenable if and only if, for any topological $K y$ Fan system $(E, H, X, G)$, the set $X_{H}$ contains a $G$-invariant element whenever it contains an $N$-invariant element.

Is there a version of (4.4) for locally compact groups? The main problem is that the $\mathrm{Ky}$ Fan system $(E, H, X, G)$ of the proof of (4.4) is not topological in general.

We now state a semigroup version of (4.4). This will prove useful in Section 6. The proof is an easier version of (4.4).

Proposition 4.8. Let $E$ be a locally convex space, $H$ be a subspace of $E, X \subset E$ and $S \subset B(E)$ be a left amenable semigroup of invertible operators such that: 
(i) the set $\mathscr{A}$ of $H$-complements in $X$ is not empty, and $s \mathscr{A} \subset \mathscr{A}$ for all $s \in S$;

(ii) $s H \subset H$ for all $s \in S$;

(iii) for each $e \in E$, the set $C_{e}=(e+H) \cap X$ is compact convex.

Then there exists $V \in \mathscr{A}$ with $s V=V$ for all $s \in S$.

\section{Finite-dimensional examples}

We give two finite-dimensional examples of Ky Fan systems. Many other natural geometric examples of $\mathrm{Ky}$ Fan systems can be constructed using group symmetries. Both examples below are reduced topological Ky Fan systems $(E, H, X, G)$; we will use the notations of (3.1) and its proof, as well as some of the notations preceding (3.1).

Example 1. Let $E=\mathbb{R}^{3}, H=\mathbb{R}^{2} \times\{0\}, G=\mathbb{T}$, the circle group realised as rotations about the $z$-axis, and $X$ be the cone $x^{2}+y^{2} \leqq z^{2}$. Let $k=(0,0,1)$. An element $T \in \mathscr{L}\left(\mathbb{R}^{3}\right)$ is in $\mathscr{P}_{H}$ if and only if $T(\lambda k+h)=\lambda\left(k+h^{T}\right)(\lambda \in \mathbb{R}, h \in H)$, where $h^{T} \in H$ is independent of $\lambda, k, h$. Obviously, $X_{H}$ is the set of lines in $X$ passing through the origin, and $C=\left\{T \in \mathscr{P}_{H}:\left|h^{T}\right| \leqq 1\right\}$. To specify $K$ (c.f. (3.1.(iii))), we take $V$ to be the $z$-axis with basis $B=\{k\}$. Identifying $H^{\mathrm{B}}$ with $H$, we have, for $h \in H, x \in G$,

$$
\left.H \cap\left(x V_{h}-k\right)=\mathbb{R}^{2} \times\{0\}\right) \cap(x(\mathbb{R}(k+h))-k),
$$

and it follows that the $G$-action on $H^{B}=H$ coincides with the given action on $H$. Now

$$
K=\left\{h \in H: P_{V_{h}} \in C\right\}=\left\{(x, y) \in \mathbb{R}^{2}: x^{2}+y^{2} \leqq 1\right\},
$$

and $G$ acts in the obvious way by rotation about the origin and has 0 as a fixed-point. The invariant line in $X$ is, of course, the $z$-axis.

Example 2. Let $E=\mathbb{R}^{3}, H=\{0\} \times \mathbb{R} \times\{0\}$ and $X=\mathbb{R} \times W$ where $W$ is the set $\left\{(y, z) \in \mathbb{R}^{2}:-|z| \leqq y \leqq|z|\right\}$. We take $G=\mathbb{Z}_{2}=\{g, e\}$, where $g$ acts on $E$ by reflecting through the $(x, y)$-plane. We take $V$ to be the $(x, z)$-plane.

Let $e_{1}=(1,0,0), e_{2}=(0,1,0)$ and $e_{3}=(0,0,1)$. Since, for any $T \in \mathscr{P}_{H}$, we have $e_{i}-$ $T e_{i} \in H$, it is obvious that the elements $T$ of $\mathscr{P}_{H}$ are given by: $T\left(e_{1}\right)=e_{1}+r_{1} e_{2}, T\left(e_{2}\right)=0$, $T\left(e_{3}\right)=e_{3}+r_{3} e_{2}$, where $\left(r_{1}, r_{3}\right) \in \mathbb{R}^{2}$. The planes in $X$ through the origin are all of the form $V_{\theta}: z=(\tan \theta) y(\pi / 4 \leqq \theta \leqq 3 \pi / 4)$. If $T \in \mathbb{P}_{H}$ is such that $T E=V_{\theta}$, then we require $\left(1, r_{1}, 0\right),\left(0, r_{3}, 1\right) \in V_{\theta}$, i.e. $r_{1}=0, r_{3}=\cot \theta$. So $C$ consists of all matrices of the form

$$
T=\left[\begin{array}{lll}
1 & 0 & 0 \\
0 & 0 & a \\
0 & 0 & 1
\end{array}\right](a \in[-1,1]) .
$$

Now $T E=\operatorname{Span}\left\{e_{1}, e_{3}+a e_{2}\right\}$, and it is obvious that the $r \in H^{2}$ for which $V_{r}=T E$ is: $r=(0, a)$ (with the obvious bases for $H$ and $V$ ). So $K=\{0\} \times[-1,1]$. It remains to 
calculate the resulting $G$-action on $H^{2}:(x, r) \rightarrow x . r$. Let $r=\left(r_{1}, r_{2}\right) \in H^{2}$. Then $g V_{r}=$ $\operatorname{Span}\left\{g\left(e_{1}+r_{1} e_{2}\right), g\left(e_{3}+r_{2} e_{2}\right)\right\}=\operatorname{Span}\left\{e_{1}+r_{1} e_{2},-e_{3}+r_{2} e_{2}\right\}$. So if $s=\left(s_{1}, s_{2}\right)=g . r$, then $\left\{s_{1} e_{2}\right\}=H \cap\left(g V_{r}-e_{1}\right)=\left\{r_{1} e_{2}\right\}$, while $\left\{s_{2} e_{2}\right\}=H \cap\left(g V_{r}-e_{3}\right)=\left\{-r_{2}\right\}$. So $g \cdot\left(r_{1}, r_{2}\right)=$ $\left(r_{1},-r_{2}\right)$. The $G$-fixed point in $K$ is, of course, $(0,0)$, which corresponds to the $G$-invariant plane $y=0$ in $\mathbb{R}^{3}$.

\section{A class of examples}

We will describe a class of semigroups of operators to which (4.8) applies. These will also yield examples of groups of operators with invariant complementary subspaces. The motivation for studying this class of examples comes from an example of Ky Fan (c.f. $[4$, p. 451]).

Let $\mathscr{H}$ be a Hilbert space, $K$ be a closed subspace of $\mathscr{H}$ and $H=K^{\perp}$. Let $Z=\{P \in B(\mathscr{H}): P K \subset H, P H=\{0\}\}$. Let $I_{K}, I_{H}$ be the orthogonal projections from $\mathscr{H}$ onto $K, H$ respectively, and let

$$
\begin{aligned}
& G=\left\{a I_{K}+b I_{H}+Q_{1}: a, b \in \mathbb{R}, a b \neq 0, Q_{1} \in Z\right\} \\
& \Sigma=\left\{k I-r\left(I_{H}+P\right): k>r \geqq 0, p \in Z,\|P\|<1\right\} .
\end{aligned}
$$

We shall see below that $G$ is a group of invertible elements of $B(\mathscr{H})$ and that $\Sigma$ is a subsemigroup of $G$. We shall apply (4.8) to the left amenable subsemigroups of $\Sigma$.

For calculation purposes, we note that $Z^{2}=\{0\}$. Further we can express $T=$ $\left(a I_{K}+b I_{H}+Q_{1}\right) \in G$ in the matrix form

$$
T=\left[\begin{array}{cc}
a & 0 \\
Q_{1} & b
\end{array}\right]
$$

where $\mathscr{H}$ is regarded as the direct sum $K \oplus H$. Similarly, $T^{\prime}=\left(k I-r\left(I_{H}+P\right)\right) \in \Sigma$ has the matrix form

$$
T^{\prime}=\left[\begin{array}{cc}
k & 0 \\
-r P & k-r
\end{array}\right] .
$$

For $\xi \in \mathscr{H}$, let $\xi=\xi_{K}+\xi_{H}$ where $\xi_{K} \in K, \xi_{H} \in H$. Let $\langle$,$\rangle be the quadratic form on \mathscr{H}$ given by: $\langle\xi, \eta\rangle=\left\langle\xi_{K}, \eta_{K}\right\rangle-\left\langle\xi_{H}, \eta_{H}\right\rangle$ (c.f. (4.5)). Let $X=\{\xi \in \mathscr{H}:\langle\xi, \xi\rangle \geqq 0\}$.

\section{Proposition 6.1.}

(i) The set $G$ is a group of invertible elements of $B(\mathscr{H})$, and $\Sigma$ is a subsemigroup of G;

(ii) $G$ is a solvable group;

(iii) $T(X) \subset X$ for all $T \in \Sigma$.

\section{Proof.}

(i) Obviously, $I \in G$. Let

$$
T=\left[\begin{array}{cc}
a & 0 \\
Q_{1} & b
\end{array}\right], S=\left[\begin{array}{cc}
c & 0 \\
R_{1} & d
\end{array}\right]
$$


be in $G$. It is elementary that $T^{-1}=(a b)^{-1}\left[\begin{array}{cc}b & 0 \\ -Q_{1} & a\end{array}\right], T S=\left[\begin{array}{cc}a c & 0 \\ c Q_{1}+b R_{1} & b d\end{array}\right]$.

Obviously, $G$ is a group.

Now let

$$
T=\left[\begin{array}{cc}
k & 0 \\
-r P & k-r
\end{array}\right], S=\left[\begin{array}{cc}
l & 0 \\
-s Q & l-s
\end{array}\right]
$$

belong to $\Sigma$. So $\|P\|,\|Q\| \leqq 1$ and $k>r \geqq 0, l>s \geqq 0$. Then

$$
T S=\left[\begin{array}{cc}
k l & 0 \\
-r l P-s(k-r) Q & (k-r)(l-s)
\end{array}\right] .
$$

Let $k^{\prime}=k l, r^{\prime}=k^{\prime}-(k-r)(l-s)$. Clearly $k^{\prime}-r^{\prime}>0$ since $0<k-r, 0<l-s$, and $r^{\prime} \geqq 0$ since $0<k-r \leqq k, 0<l-s \leqq l$. Also, $-r l P-s(k-r) Q=-r^{\prime} Q^{\prime}$, where $Q^{\prime} \in Z$ has norm $\leqq 1$ since $Q^{\prime}$ is a convex combination of $P, Q$. So $\Sigma$ is a semigroup.

(ii) Let $T, S \in G$. Then $T^{-1} S^{-1} T S$ is of the form

$$
\left[\begin{array}{ll}
1 & 0 \\
Q^{\prime} & 1
\end{array}\right]
$$

and so therefore are all the elements in the commutator subgroup $G^{\prime}$ of $G$. The elements of $G^{\prime}$ commute so that $G$ is solvable of rank 2 .

(iii) Let $T \in \Sigma$ be as in the above proof: so $k>r \geqq 0,\|P\| \leqq 1$. Let $\xi \in X$. Then $\left\|\xi_{K}\right\| \geqq\left\|\xi_{H}\right\|$, and

$$
T \xi=k \xi_{K}+\left[(k-r) \xi_{H}-r P \xi_{K}\right]
$$

So

$$
\begin{aligned}
\langle T \xi, T \xi\rangle= & k^{2}\left\|\xi_{K}\right\|^{2}-\left\|(k-r) \xi_{H}-r P \xi_{K}\right\|^{2} \\
= & k^{2}\left\|\xi_{K}\right\|^{2}-\left[(k-r)^{2}\left\|\xi_{H}\right\|^{2}+r^{2}\left\|P \xi_{K}\right\|^{2}-2(k-r) r \operatorname{Re}\left(\xi_{H}, P \xi_{K}\right)\right] \\
\geqq & {\left[(k-r)^{2}+2(k-r) r+r^{2}\right]\left\|\xi_{K}\right\|^{2}-\left[(k-r)^{2}\left\|\xi_{H}\right\|^{2}+r^{2}\left\|\xi_{K}\right\|^{2}\right] } \\
& -2(k-r) r\left\|\xi_{H}\right\|\left\|\xi_{K}\right\| \\
= & (k-r)^{2}\left(\left\|\xi_{K}\right\|^{2}-\left\|\xi_{H}\right\|^{2}\right)+2(k-r) r\left\|\xi_{K}\right\|\left(\left\|\xi_{K}\right\|-\left\|\xi_{H}\right\|\right) \\
\geqq &
\end{aligned}
$$

So $T X \subset X$. 
Proposition 6.2. Let $G, \Sigma, X, \mathscr{H}, K, H$ be as above, and $\Sigma^{\prime}$ be a left amenable subsemigroup of $\Sigma$. Then there exists a positive, $\Sigma$ '-invariant, topological complement for $H$ in $\mathscr{H}$.

Proof. Clearly, $H$ is $G$-invariant and so $\Sigma^{\prime}$-invariant. The space $K$ belongs to set $\mathscr{A}$ of $H$-complements in $X$, and using (6.1.(iii)), we have $s \mathscr{A} \subset \mathscr{A}$ for all $s \in \Sigma^{\prime}$. The condition (4.8.(iii)) is satisfied (c.f. (4.4), (4.5)) (with $\mathscr{H}$ given the weak topology). So there exists an $H$-complement $V \in \mathscr{A}$ with $s V \subset V$ for all $s \in \Sigma^{\prime}$. Now $X$ is norm closed in $\mathscr{H}$, and so $V^{-} \subset X$. Since $V^{-} \cap H \subset H \cap X=\{0\}$, we have $V=V^{-}$, and $V$ is a topological complement for $H$ in $\mathscr{H}$ (for the norm topology).

Notes. It follows from (6.2) that a subgroup of $G$ generated by a left amenable subsemigroup of $\Sigma$ will also leave invariant some positive topological $H$-complement. The question of which subsemigroups of $\Sigma$ are left amenable raises some interesting issues which the authors have been unable to resolve. We will now briefly discuss these.

Since any abelian semigroup is amenable, the above Proposition applies to all such subsemigroups of $\Sigma$-in particular, to singly-generated subsemigroups of $\Sigma$. If $\Sigma^{\prime}$ is a subsemigroup of $\Sigma$, then the subgroup $G^{\prime}$ of $G$ generated by $\Sigma^{\prime}$ is solvable (c.f. (6.1.(ii))) and so amenable. However, it is well known that there are solvable groups (e.g. the " $a x+b$ "-group) containing non-amenable subsemigroups. (We will produce an example of this below.) The semigroup $\Sigma^{\prime}$ will be left amenable if either of the two conditions holds:

(a) $s^{-1} \Sigma^{\prime} s \subset \Sigma^{\prime}$

(b) $\Sigma^{\prime}$ is a semidirect product $\Sigma_{1} \times_{\rho} \Sigma_{2}$ of left amenable semigroups $\Sigma_{1}, \Sigma_{2}$ with $\rho(t)$ surjective for all $t \in \Sigma_{2}$. (These results are due to Day and Klawe-a full account of these is given in Paterson [15].) Unfortunately, we have been unable to use either of these criteria to produce non-abelian amenable subsemigroups of $\Sigma^{\prime}$.

In fact, the simple argument below suggests that left amenable subsemigroups of $\Sigma$ are rather sparse in general. Let $\mathscr{M}=\mathbb{C}^{2}$ with $K=\mathbb{C} \oplus\{0\}, H=\{0\} \oplus \mathbb{C}$. Then the elements $T$ of $\Sigma$ are of the form

$$
\left[\begin{array}{cc}
k & 0 \\
-r t & k-r
\end{array}\right] \quad(k>r \geqq 0, t \in \mathbb{C},|t| \leqq 1) .
$$

Suppose that $\Sigma^{\prime} \subset \Sigma$ is left amenable. By (6.2), the elements of $\Sigma^{\prime}$ have a common eigenvalue $\mathbf{v} \notin H$. If $\mathbf{v} \in K$, then the elements of $\Sigma^{\prime}$ are diagonal, and $\Sigma^{\prime}$ is abelian. If $v \notin K$, then we can suppose $\mathbf{v}=\left[\begin{array}{ll}a & 1\end{array}\right]^{t}$ for some $a \in \mathbb{C} \sim\{0\}$. An elementary eigenvalue calculation shows that $T \in \Sigma^{\prime}$ is either of the form $k I(k>0)$ or of the form

$$
\left[\begin{array}{cc}
k & 0 \\
r a^{-1} & k-r
\end{array}\right]
$$

(In particular, $|a| \geqq 1$.) It follows that $\Sigma^{\prime}$ is abelian and we have "left amenable=abelian" 
for subsemigroups of $\Sigma$. Since $\Sigma$ is not abelian, $\Sigma$ is an example of a non-amenable subsemigroup of a solvable group.

\section{REFERENCES}

1. W. G. Dixon, Special Relativity (Cambridge University Press, 1978).

2. P. EYMARD, Moyennes invariantes et représentations unitaires (Lecture Notes in Mathematics, 300, Springer-Verlag, Berlin, 1972).

3. K. FAN, Invariant subspaces of certain linear operators, Bull. Amer. Math. Soc. 69 (1963), 773-777.

4. K. FAN, Invariant cross-sections and invariant linear subspaces, Israel J. Math. 2 (1964), 19-26.

5. K. FAN, Invariant subspaces for a semigroup of linear operators, Indag. Math. 27 (1965), 447-451.

6. F. P. Greenleaf, Invariant means on topological groups (Van Nostrand Math. Stud., Princeton, N.J., 1969).

7. I. S. Ionvidov, Unitary operators in a space with indefinite metric, Zapiski Har'kov. Mat. Obšč. 21 (1949), 79-86.

8. G. Kӧтн, Topological vector spaces I (Springer-Verlag, Berlin-Heidelberg-New York, 1969).

9. M. G. KREIN, On an application of the fixed-point principle in the theory of linear transformations of spaces with an indefinite metric, Amer. Math. Soc. Transl. Ser. 2, 1 (1955), 27-35.

10. A. T. LAU, Finite-dimensional invariant subspaces for a semigroup of linear operators, $J$. Math. Anal. Appl. 97 (1983), 374-379.

11. A. T. LAU and J. C. S. WONG, Invariant subspaces for algebras of linear operators and amenable locally compact groups, 1986, preprint.

12. A. T. LAU and J. C. S. WONG, Finite-dimensional invariant subspaces for measurable semigroups of linear operators, J. Math. Anal. Appl. 127 (1987), 548-558.

13. M. A. Nalmark, Commutative unitary operators on a $\pi_{k}$-space, Soviet Math. 4 (1963) 543-545.

14. M. A. Nalmark, On commuting unitary operators in spaces with indefinite metric, Acta Sci. Math. (Szeged) 24 (1963), 177-189.

15. A. L. T. Paterson, Amenability (Mathematical Surveys and Monographs, American Mathematical Society, 1988).

16. J.-P. PIER, Amenable locally compact groups (John Wiley and Sons, New York, 1984).

17. L. S. Pontruagin, Hermitian operators in spaces with indefinite metric, Izv. Akad. Nauk SSSR. Ser. Mat. 149 (1963), 1261-1263.

UnIVERSity OF AlBerta

Alberta

Canada

University of Calgary

Calgary

CANADA
University of Aberdeen

AberdeEn

SCOTLAND

Current address

UNIVERSITY OF MisSISSIPPI

MS 38677 